\title{
THE CENTRE OF A HEREDITARY LOCAL RING
}

\author{
by D. G. NORTHCOTT \\ (Received 10 January, 1961)
}

The purpose of this note is to establish the following

THEOREM. The centre of a (left) hereditary local ring is either a field or a one-dimensional regular local ring.

Before starting the proof, it is necessary to explain the terminology. A ring $R$ with an identity element is called a left local ring if the elements of $R$ which do not have left inverses form a left ideal $I$. In these circumstances (see [1, Proposition 2.1, p. 147]), $I$ is necessarily a two-sided ideal and it consists precisely of all the elements of $R$ which do not have right inverses. Furthermore, every element of $R$ which is not in $I$ possesses a two-sided inverse. Thus there is, in fact, no difference between a left local ring and a right local ring and therefore one speaks simply of a local ring. In addition, $I$ contains every proper left ideal and every proper right ideal. We may therefore describe $I$ simply as the maximal ideal of $R$.

A ring $R$ is called left hereditary if every left ideal is a projective $R$-module. Now, by a theorem due to Kaplansky [2, Theorem 2, p. 374], every projective module with respect to a local ring is free. Accordingly, if $R$ is a left hereditary local ring, then every left ideal possesses a base.

From now on let $R$ be a (left) hereditary local ring and let $I$ be its maximal ideal. Further, let $Q$ be the centre of $R$ and assume that $Q$ is not a field. We contend that $Q$ is a onedimensional regular local ring. This includes the assertion that $Q$ is Noetherian.

Put $J=Q \cap I$, so that $J$ is a proper ideal of $Q$. Suppose that $q \in Q, q \notin J$. Then $q$ has an inverse $x$ in $R$ and one verifies immediately that $x \in Q$. It follows that $Q$ is a local ring and $J$ is its maximal ideal. Since $Q$ is not a field, $J \neq(0)$.

Now consider $R J$. This is a left ideal of $R$ and therefore it possesses an $R$-base. Furthermore, since $R J \neq(0)$ and $I$ is a two-sided ideal, $R J$ is not contained in $I(R J)$; consequently we can find $\gamma \in J$ such that $\gamma \notin I(R J)$. If now $\gamma$ is expressed in terms of the base of $R J$, at least one of the coefficients will be a unit in $R$. It follows that one of the base elements can be replaced by $\gamma$ in such a way that we still get a base. But $\gamma$ is now the only element in the new base. For suppose that $u$ were a different base element. Then, since $\gamma$ belongs to the centre of $R, u \gamma+(-\gamma) u=0$, and this gives a contradiction. This shows that $R J=R \gamma$. Furthermore, since $\gamma$ is a base for this ideal, $\gamma$ is not a zero-divisor in $R$.

Let $q \in R \gamma \cap Q$; then $q=z \gamma$ with $z \in R$. If now $y$ is any element of $R$,

$$
z y \gamma=z \gamma y=q y=y q=y z \gamma,
$$

whence $z y=y z$ because $\gamma$ is not a zero-divisor. This shows that $z \in Q$ and we conclude that $R \gamma \cap Q \subseteq Q \gamma$. But $R \gamma \cap Q=R J \cap Q \supseteq J$ and therefore $J=Q$.

Next put

$$
A=\bigcap_{=1}^{\infty} Q \gamma^{n}
$$


so that $A$ is a $Q$-ideal. Since $\gamma$ is not a zero-divisor, we see that $\gamma A=A$. Remembering that $\gamma$ is in the centre of $R$, we obtain

$$
R A=\gamma(R A) \subseteq I(R A) .
$$

But $R A$, being a left ideal of $R$, possesses an $R$-base and now the above relation shows that $R A$ must be the zero ideal. Thus $A=(0)$ and hence

$$
\bigcap_{n=1}^{\infty} Q \gamma^{n}=(0) \text {. }
$$

Finally, let $B$ be any non-zero ideal of $Q$. If $m$ is the largest integer such that $B \subseteq Q \gamma^{m}$, then we see easily that $B=Q \gamma^{m}$. Thus $Q$ is a commutative local ring, which is not a field, in which every ideal is a principal ideal. Since the maximal ideal contains at least one element which is not a zero-divisor, this completes the proof.

\section{REFERENCES}

1. H. Cartan and S. Eilenberg, Homological algebra (Princeton, 1956).

2. I. Kaplansky, Projective modules, Ann. of Math. 68 (1958), 372-377.

\section{THE UNIVERSITY}

SHEFFIELD 